Note: Scale-free center-of-mass displacement correlations in polymer films without topological constraints and momentum conservation

J. P. Wittmer, N. Schulmann, P. Polińska, and J. Baschnagel

Citation: The Journal of Chemical Physics 135, 186101 (2011); doi: 10.1063/1.3662132

View online: https://doi.org/10.1063/1.3662132

View Table of Contents: http://aip.scitation.org/toc/jcp/135/18

Published by the American Institute of Physics 


\title{
Note: Scale-free center-of-mass displacement correlations in polymer films without topological constraints and momentum conservation
}

\author{
J. P. Wittmer, ${ }^{\text {a) }}$ N. Schulmann, P. Polińska, and J. Baschnagel \\ Institut Charles Sadron, Université de Strasbourg, CNRS, 23 rue du Loess, 67034 Strasbourg Cedex, France
}

(Received 15 September 2011; accepted 27 October 2011; published online 14 November 2011)

[doi:10.1063/1.3662132]

Polymer melts without topological constraints are believed to be described by the Rouse model, ${ }^{1}$ i.e., the random forces from the bath acting on the center-of-mass $(\mathrm{CM}) \underline{r}_{\mathrm{N}}(t)$ of a reference chain of length $N$ are supposed to be uncorrelated. For the corresponding CM mean-square displacement (MSD) $h_{\mathrm{N}}(t)$, this implies

$$
h_{\mathrm{N}}(t) \equiv\left\langle\left(\underline{r}_{\mathrm{N}}(t)-\underline{r}_{\mathrm{N}}(0)\right)^{2}\right\rangle=2 d D_{\mathrm{N}} t
$$

for all times $t$ with $d$ being the spatial dimension, $D_{\mathrm{N}} \approx b^{2} W / N$ being the self-diffusion coefficient, and $b$ being the effective bond length. ${ }^{1}$ The monomer mobility $W$ may be obtained by fitting the monomer MSD

$$
h(t) \equiv\left\langle(\underline{r}(t)-\underline{r}(0))^{2}\right\rangle=b^{2}(W t)^{2 \alpha} \quad \text { with } \alpha=1 / 4
$$

$(\underline{r}(t)$ being the monomer position) for times smaller than the relaxation time $T_{\mathrm{N}} \approx N^{2} / W{ }^{1}$ In fact, correlated (colored) forces have been observed both experimentally and numerically as reviewed in Ref. 2 . These colored forces are best captured in a computer simulation using the "velocity correlation function" $(\mathrm{VCF})^{3}$

$$
C_{\mathrm{N}}(t) \equiv\left\langle\frac{\underline{u}(t)}{\delta t} \cdot \frac{\underline{u}(0)}{\delta t}\right\rangle \approx \frac{1}{2} \frac{\partial^{2} h_{\mathrm{N}}(t)}{\partial t^{2}} \sim \delta t^{0} \quad \text { for } t \gg \delta t
$$

which measures directly the curvature of $h_{\mathrm{N}}(t)$ with respect to time with $\underline{u}(t)=\underline{r}_{\mathrm{N}}(t+\delta t)-\underline{r}_{\mathrm{N}}(t)$ being the CM displacement for a time window $\delta t$. Using simple scaling arguments it has recently been suggested ${ }^{3}$ that an interplay of melt incompressibility and chain connectivity implies a negative algebraic decay of the VCF

$$
C_{\mathrm{N}}(t)=-c \frac{D_{\mathrm{N}} W}{b^{d} \rho}(W t)^{-\omega} \quad \text { for } t \ll T_{\mathrm{N}} \approx N^{2} / W
$$

with $c$ being an empirical amplitude, $\rho$ being the monomer density, and $\omega=(2+d) \alpha$ being the exponent characterizing the colored forces. This scaling has been demonstrated numerically by means of Monte Carlo (MC) simulations for three-dimensional (3D) melts where $\omega=5 / 4 .{ }^{3}$ The aim of this note is to show that Eq. (4) holds also in effectively twodimensional (2D) polymer films.

As in Ref. 3 we use a version of the bond-fluctuation model (BFM) with finite excluded volume penalty $\varepsilon$ and topology violating local moves to the 26 neighboring lattice sites. Length scales are given in units of the lattice constant, time in Monte Carlo steps. The chains are confined to a slit of width $H=4$ between two parallel repulsive walls following Ref. 4. All properties discussed refer to their 2D projection parallel to the walls. Working at the 3D volume fraction $\phi=0.5$ of occupied lattice sites, ${ }^{4}$ the relevant $2 \mathrm{D}$ number density is thus $\rho=(\phi / 8) H=0.25$. The systems are effectively $d=2$ dimensional since the width of the slit is much smaller than the typical chain size. For the overlap penalty $\varepsilon=10$ (in units of $k_{\mathrm{B}} T$ ) presented here the static properties are similar to Ref. 4 where $\varepsilon=\infty$. Note that the monomers can still cross each other in the direction perpendicular to the walls. The chains are thus "self-avoiding trails," 5 i.e., they adopt logarithmically swollen configurations. ${ }^{4,5}$ Since $R_{\mathrm{N}}^{2} / N$ diverges for large $N$ (albeit very weakly), the operational definition of the effective 2D bond length $b$ is a delicate issue and the projected effective bond length of the bulk, $b \equiv 3.24 \sqrt{2 / 3} \approx 2.65$, is used as the reference. Note that the scaling of the dynamical properties discussed below does not depend on this specific value.

It is important to stress that due to the use of finite overlap penalty and topology non-conserving MC moves, the dynamics remains to leading order of Rouse-type as can be seen from the MSDs presented in Fig. 1. The monomer MSD $h(t)$ (open symbols) compares well over several orders of magnitude to Eq. (2) indicated by the dashed line. Although it is

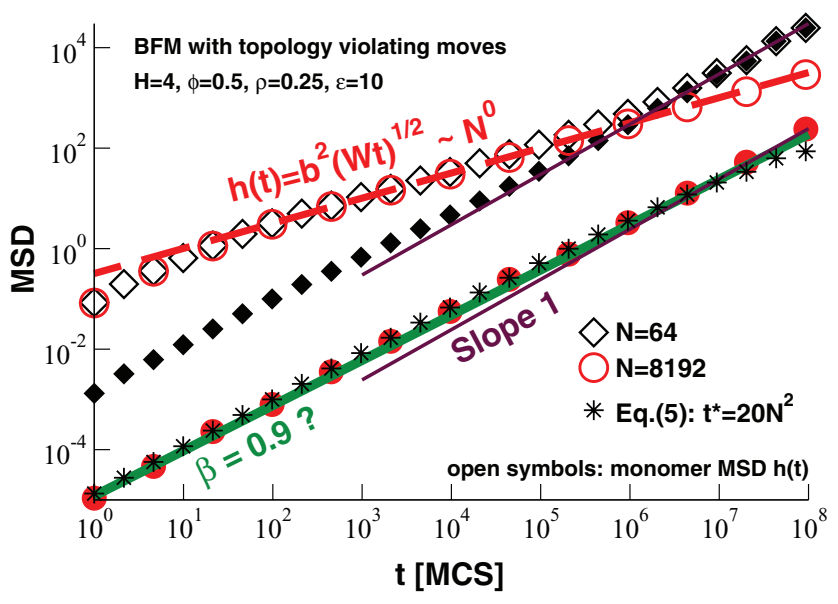

FIG. 1. Various MSDs for polymer melts confined to ultrathin films of width $H=4$ computed using the BFM algorithm with topology violating local MC moves. ${ }^{3}$ The open symbols represent the monomer MSD $h(t)$ which compare well to the Rouse prediction (dashed line). Systematic deviations from the Rouse model are revealed for the CM MSD $h_{\mathrm{N}}(t)$ (filled symbols) as emphasized by the effective power-law exponent $\beta=0.9$ (bold line). The stars correspond to Eq. (5) with $c=1$ and $t^{*}=20 N^{2}$ for $N=8192$. 


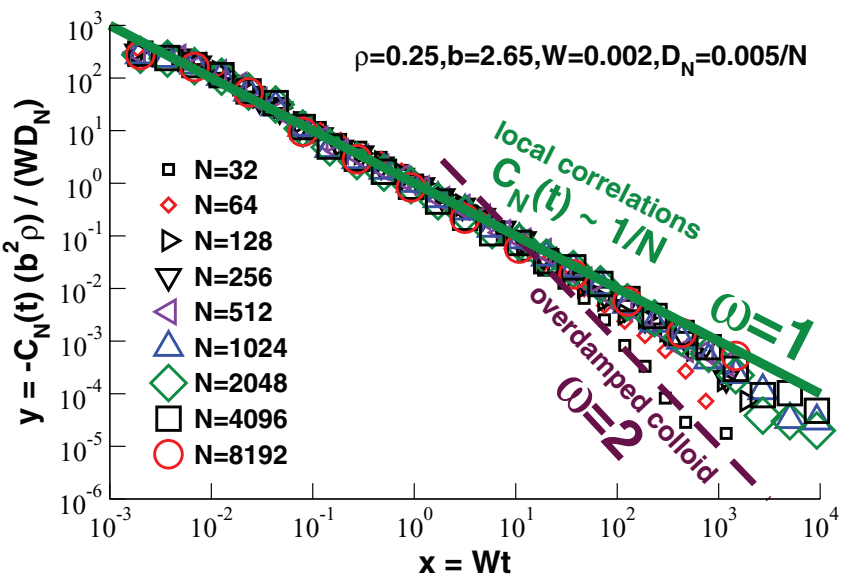

FIG. 2. Scaling plot of the directly computed displacement correlation function $C_{\mathrm{N}}(t)$ for a large range of chain lengths $N$ (open symbols) using the parameters $\rho, b, W$, and $D_{\mathrm{N}} \sim 1 / N$ indicated in the plot. The data collapse for $t \ll T_{\mathrm{N}}$ confirms that $C_{\mathrm{N}}(t) \sim 1 / N$. The corresponding time exponent $\omega$ $=1$ (bold line) is observed over up to five orders of magnitude in time. The exponent $\omega=2$ for overdamped colloids expected for $t \gg T_{\mathrm{N}}$ is indicated by the dashed line.

likely that this scaling does not hold in a strict sense due to the mentioned logarithmic swelling of the chains, deviationsif they exist-are apparently of higher order and irrelevant on the logarithmic scales we focus on. As can be seen for $N$ $=64$, the monomers diffuse again freely (thin solid line) for times $t \gg T_{\mathrm{N}}$. Please note that it was not the aim of the present work to sample for our larger chains $(N>2000)$ over the huge times needed to make this regime accessible. The short-time behavior, Eq. (2), can be used to determine $W \approx 0.002$ for the effective local mobility and to predict a self-diffusion coefficient $D_{\mathrm{N}} \approx 0.005 / N$ using the Rouse model. ${ }^{3}$ Systematic deviations from the Rouse scaling are revealed, however, for the CM MSD $h_{\mathrm{N}}(t)$ (filled symbols) as emphasized by the effective exponent $\beta=0.9$ (bold line). At variance to the $3 \mathrm{D} \mathrm{bulk}{ }^{3}$ deviations are visible up to a time $t^{*}$ scaling as the chain relaxation time $T_{\mathrm{N}}$. (It is thus only possible to confirm the given value of $D_{\mathrm{N}}$ from the long-time plateau of $h_{\mathrm{N}}(t) / 2 d t$ for chains up to $N=1024$.)

A more precise characterization of the colored forces acting on the chains is achieved by means of the VCF $C_{\mathrm{N}}(t)$ computed directly as described in Ref. 3 . The rescaled data presented in Fig. 2 confirm the predicted algebraic decay, Eq. (4), with an exponent $\omega=1$ and an empirical amplitude $c \approx 1$ (bold line). Note that the scaling implies $C_{\mathrm{N}}(t) \sim 1 / N$, i.e., the correlations are due to $\sim N$ local independent events as for 3D melts. The large time behavior for $t \gg T_{\mathrm{N}}$ can be seen for the shorter chains which approach the exponent $\omega$ $=(2+d) / 2=2$ (dashed line) expected for overdamped colloids in incompressible solutions. ${ }^{3,6}$ Using Eq. (3) it follows from Eq. (4) that

$$
h_{\mathrm{N}}(t)=4 D_{\mathrm{N}} t\left(1+\frac{c}{2 b^{2} \rho} \ln \left(t^{*} / t\right)\right) \text { for } t \ll T_{\mathrm{N}}
$$

with $t^{*}$ being an integration constant. Please note that the logarithmic contribution to Eq. (5) dominates for $t \ll t^{*}$. While in 3D we have $t^{*} \sim N^{0} \ll T_{\mathrm{N}}{ }^{3}$ it turns out that for thin films $t^{*} \approx 20 N^{2} \approx T_{\mathrm{N}} / 18$ yields a good fit for all chain lengths we have sampled as can be seen for $N=8192$ in Fig. 1 (stars).

In summary, we have presented here computational work on the displacement correlations in thin polymer films without topological constraints and momentum conservation using a well-known lattice MC algorithm. ${ }^{3,7}$ As anticipated by the scaling result suggested recently for $3 \mathrm{D}$ melts, ${ }^{3}$ it is shown here for effectively $2 \mathrm{D}$ systems that the VCF $C_{\mathrm{N}}(t)$ reveals a negative algebraic decay with a power-law exponent $\omega=(2$ $+d) / 4=1$. This implies a logarithmic correction to the CM MSD. Our MC approach corresponding to a strongly overdamped melt should be relevant to most experimental setups due to the strong frictional forces from the walls. Obviously, qualitatively different correlations are to be expected if hydrodynamic interactions matter-as is the case for molecular dynamics simulations of a bead-spring model with a weak Langevin thermostat ${ }^{8}$ —or if the chains are confined to strictly 2D layers due to the ensuing compactness and surface fractality of the chains. 5,9

\footnotetext{
${ }^{a)}$ Electronic mail: joachim.wittmer@ics-cnrs.unistra.fr.

${ }^{1}$ M. Doi and S. F. Edwards, The Theory of Polymer Dynamics (Clarendon, Oxford, 1986).

${ }^{2}$ W. Paul and G. Smith, Rep. Prog. Phys. 67, 1117 (2004).

${ }^{3}$ J. Wittmer, P. Polińska, A. Cavallo, H. Meyer, J. Farago, A. Johner, and J. Baschnagel, J. Chem. Phys. 134, 234901 (2011).

${ }^{4}$ A. Cavallo, M. Müller, J. P. Wittmer, A. Johner, and K. Binder, J. Phys.: Condens. Matter 17, S1697 (2005).

${ }^{5}$ A. N. Semenov and A. Johner, Eur. Phys. J. E 12, 469 (2003).

${ }^{6}$ J. K. G. Dhont, An Introduction to Dynamics of Colloids (Elsevier, Amsterdam, 1996).

${ }^{7}$ J. P. Wittmer, A. Cavallo, T. Kreer, J. Baschnagel, and A. Johner, J. Chem. Phys. 131, 064901 (2009).

${ }^{8}$ J. Farago, H. Meyer, and A. N. Semenov, Phys. Rev. Lett. 107, 178301 (2011).

${ }^{9}$ J. P. Wittmer, H. Meyer, A. Johner, T. Kreer, and J. Baschnagel, Phys. Rev.
} Lett. 105, 037802 (2010). 